\section{Attention on Infection Following Transcatheter Aortic Valve Implantation}

To the Editor-We have read with interest the study by Shi et $\mathrm{al}^{1}$ on the incidence and risk factors for infection following transcatheter aortic valve implantation (TAVI). In their report, 35 of 253 patients had infections during the 30 days after the procedure; chronic obstructive pulmonary disease, postprocedural hemorrhage, and procedure-related stroke were identified as variables associated with the development of infection. Although infection has been recognized as one of the most common complications after surgery, its incidence, etiology, and impact following TAVI are not well defined yet. This study, with clear classification of infection types and determination of risk factors, certainly sheds some light on our understanding of this less discussed complication.

Compared with issues such as paravalvular leak, conduction disturbances, or stroke after TAVI, infections seem to be somewhat neglected. Although TAVI is minimally invasive, TAVI candidates usually have predisposing factors for infection, including age; poor pulmonary, renal, and immune function; diabetes mellitus; and need for ventilation and central venous access. Chronic obstructive pulmonary disease was associated with post-TAVI infection in both their univariate and multivariate analyses. In our group's previous systematic review, ${ }^{2}$ infection/sepsis was the most common cause of death both within and beyond 30 days of TAVI in the pooled analysis. In this study, ${ }^{1}$ the authors found $8(22.9 \%)$ of those who developed a post-TAVI infection died whereas only $4(1.8 \%)$ of those patients who did not have an infection died $(P<.001)$. Moreover, Tirado-Conte et $\mathrm{al}^{3}$ also recently contributed research on this issue. During a median follow-up of 21 months, 51 of 303 patients experienced in-hospital infections after TAVI, with respiratory and urinary tract infections being most frequent. Patients with infections also had increased mortality during follow-up, as well as a longer hospital stay and higher readmission rate. These findings altogether demonstrate that infections play a major adverse role in postprocedural management of TAVI recipients.

Respiratory and urinary tract infections were the most frequent type of infections in this study, ${ }^{1}$ whereas postprocedural hemorrhage requiring transfusion was found to be significantly associated with an increased infection risk. Some of these risk factors are potentially modifiable and emphasize the importance of implementing a standardized postprocedural protocol to offer our patients a quick and smooth recovery. Two recently published articles ${ }^{4,5}$ have recommended early removal of Foley catheters and other hemodynamic lines if possible. Expedited transfer from the critical care area to a cardiac step-down unit and criteria-driven early discharge were also encouraged in suitable patients. Theoretically, infections can be expected to decrease once predisposing factors are modified by those enhanced recovery pathways. However, future randomized trials are needed to verify whether these proposed measures can lower infection rates or not.

\section{ACKNOWLEDGMENTS}

Financial support. None reported.

Potential conflicts of interest. Both authors report no conflicts of interest relevant to this article.

Tian-Yuan Xiong, MD; Mao Chen, MD, PhD

Affiliations: Department of Cardiology, West China Hospital, Sichuan University, China.

Address correspondence to Mao Chen, MD, PhD, Department of Cardiology, West China Hospital, Sichuan University, 37 Guoxue Alley, Chengdu, 610041, PR China (hmaochen@vip.sina.com).

Infect Control Hosp Epidemiol 2016;37:1392

(C) 2016 by The Society for Healthcare Epidemiology of America.. All rights reserved. 0899-823X/2016/3711-0021. DOI: 10.1017/ice.2016.190

\section{REFERENCES}

1. Shi Y, Wijeysundera HC, Fremes SE, Simor AE. Incidence and risk factors for infection following transcatheter aortic valve implantation [published online June 20, 2016]. Infect Control Hosp Epidemiol 2016:1-4.

2. Xiong T-Y, Liao Y-B, Zhao Z-G, et al. Causes of death following transcatheter aortic valve replacement: a systematic review and meta-analysis. J Am Heart Assoc 2015;4:e002096.

3. Tirado-Conte G, Freitas-Ferraz AB, Nombela-Franco L, et al. Incidence, causes, and impact of in-hospital infections after transcatheter aortic valve implantation. Am J Cardiol 2016;118: 403-409.

4. Lauck SB, Wood DA, Baumbusch J, et al. Vancouver transcatheter aortic valve replacement clinical pathway: minimalist approach, standardized care, and discharge criteria to reduce length of stay. Circ Cardiovasc Qual Outcomes 2016;9:312-321.

5. Sola M, Ramm CJ, Kolarczyk LM, et al. Application of a multidisciplinary enhanced recovery after surgery pathway to improve patient outcomes after transcatheter aortic valve implantation. Am J Cardiol 2016;118:418-423.

\section{Comment on: The Carbapenemase Menace: Do Dual Mechanisms Code for More Resistance?}

To the Editor-We read with interest the letter by Kazi et $\mathrm{al}^{1}$ in which the authors reflect on the question of probable higher carbapenem minimum inhibitory concentrations (MICs) when dual carbapenem resistance mechanisms coexist. 
Greece was the first country where double carbapenemaseproducing Klebsiella pneumoniae (CPKP) were detected in 2009 . $^{2}$ Since then, they have reached approximately $5 \%$ of CPKPs isolated in Greek hospitals. ${ }^{3}$ These double CPKPs commonly bear KPC-2 plus VIM-1 carbapenemases. More recently, NDM-1-producing K. pneumoniae have also emerged in Greece. ${ }^{4}$

To further investigate the aforementioned question with a different carbapenemase combination than that used by Kazi et al, we performed the imipenem E-test (bioMérieux, Marcy l'Etoile, France) in 4 groups of nonduplicate CPKPs isolated from various clinical samples from patients hospitalized in AHEPA University Hospital of Thessaloniki. Group A consisted of 20 KPC-2-producers; group B consisted of 20 VIM-1producers; group C consisted of $20 \mathrm{NDM}-1$-producers; and group D consisted of $20 \mathrm{KPC}-2+\mathrm{VIM}-1$-producers. Bacterial identification and initial antimicrobial susceptibility testing of the isolates was performed using the VITEK2 automated system (bioMérieux, Marcy l' Étoile, France), and the presence of carbapenemase-encoding genes was determined by phenotypic tests followed by polymerase chain reaction assays with specific primers to identify the presence of carbapenemases (ie, $b l a_{\mathrm{VIM}}, b l a_{\mathrm{KPC}}$, or $\left.b l a_{\mathrm{NDM}}\right) .^{5,6}$

The imipenem MICs for the 3 groups are shown in Table 1. Moreover, we calculated the geometric mean of the MIC values for each study group as the $n$th root $(\mathrm{n}=$ the number of isolates) of the product of the MIC values using a geometric mean calculator. (A value of 64 was used conventionally for isolates with MIC $>32 \mu \mathrm{g} / \mathrm{mL}$.) Groups A and C had lower geometric means (49.496 and 47.130, respectively) compared to the conventional 64.000 of $\mathrm{B}$ and $\mathrm{D}$.

Apparently, when studying large groups of carbapenemaseproducing bacteria, more mechanisms may code for more resistance. This finding is in accord with the results of Kazi et al and with our previous study on carbapenem-resistant Pseudomonas aeruginosa, in which various mechanisms (eg, the VIM-2 carbapenemase, efflux pump over-expression, loss of the OprD porin, and the presence of inducible AmpC beta-lactamases) where taken into account. ${ }^{7}$

For single isolates, however, this cannot be considered the rule. Kazi et al found a Klebsiella spp. coproducing NDM-1 and

TABLE 1. Relation of Minimum Inhibitory Concentrations of Imipenem to Carbapenemase Presence in K. pneumoniae Isolates

\begin{tabular}{lccrrrr}
\hline & \multicolumn{6}{c}{ Imipenem MIC $(\mu \mathrm{g} / \mathrm{mL})$} \\
\hline Mechanism $(\mathrm{N}=80)$ & 6 & 8 & 16 & 24 & 32 & $>32$ \\
KPC-2 $(\mathrm{N}=20)$ & $\ldots$ & 1 & 1 & 1 & 1 & 16 \\
VIM-1 $(\mathrm{N}=20)$ & $\ldots$ & $\ldots$ & $\ldots$ & $\ldots$ & $\ldots$ & 20 \\
NDM-1 $(\mathrm{N}=20)$ & 1 & 1 & $\ldots$ & 1 & 1 & 16 \\
KPC-2+VIM-1 $(\mathrm{N}=20)$ & $\ldots$ & $\ldots$ & $\ldots$ & $\ldots$ & $\ldots$ & 20 \\
\hline
\end{tabular}

NOTE. MIC, minimum inhibitory concentration; KPC-2, Klebsiella pneumoniae carbapenemase-2; VIM-1, Verona integron-encoded metallo- $\beta$-lactamase-1; NDM-1, New Delhi metallo- $\beta$-lactamase-1.
VIM-1 with carbapenem MICs $<2 \mu \mathrm{g} / \mathrm{mL}$. Lower MICs in double-carbapenemase producers than those observed commonly in single-carbapenemase-producing isolates have also been observed elsewhere. ${ }^{8}$ These findings can be attributed to the variable expression levels of the carbapenemaseencoding genes within bacterial cells and to the different hydrolytic potential of each enzymatic type. It is known, for example, that OXA-type carbapenemases have a lower potential of carbapenem hydrolysis than the metallobeta-lactamases and Klebsiella pneumoniae carbapenemases. ${ }^{9}$

Interestingly, in our study, differences were not detected between groups $\mathrm{B}$ and $\mathrm{D}$, probably because the presence of VIM-1 was enough to raise the imipenem MICs over $32 \mu \mathrm{g} / \mathrm{mL}$ for all 20 isolates of group B. Differences between these 2 groups may exist at higher MIC levels; however, these differences would be not relevant in clinical practice because the use of carbapenems, alone or in combination with other antibiotics, is not applicable with MIC values $>32 \mu \mathrm{g} / \mathrm{mL}$.

Overall, we agree with Kazi et al that the emergence of double-carbapenemase-producing bacteria is alarming and further confines the already limited treatment options.

\section{ACKNOWLEDGMENTS}

Financial support: No financial support was provided relevant to this article.

Potential conflicts of interest: All authors report no conflicts of interest relevant to this article.

\section{Georgios Meletis, MD, MSc, $\mathrm{PhD}{ }^{1}$ Efthymia Protonotariou, $\mathrm{PhD} ;{ }^{2}$ Dimitra Papadopoulou, MSc; Lemonia Skoura, $\mathrm{PhD}^{2}$}

\footnotetext{
Affiliations: 1. Labnet Laboratories, Thessaloniki, Greece; 2. Department of Microbiology, AHEPA University Hospital, Thessaloniki, Greece.

Address correspondence to Georgios Meletis MD, MSc, PhD, Agiou Dimitriou str. 161, 53337, Thessaloniki, Greece (meletisg@hotmail.com). Infect Control Hosp Epidemiol 2016;37:1392-1394

(c) 2016 by The Society for Healthcare Epidemiology of America. All rights reserved. 0899-823X/2016/3711-0022. DOI: 10.1017/ice.2016.197
}

\section{REFERENCES}

1. Kazi M, Shetty A, Rodrigues C. The carbapenemase menace: do dual mechanisms code for more resistance? Infect Control Hosp Epidemiol 2015;36:116-117.

2. Meletis G, Tzampaz E, Protonotariou E, Sofianou D. Emergence of Klebsiella pneumoniae carrying blaVIM and blaKPC genes. Hippokratia 2010;14:139.

3. Giakkoupi P, Papagiannitsis CC, Miriagou V, et al. An update of the evolving epidemic of blaKPC-2-carrying Klebsiella pneumoniae in Greece (2009-10). J Antimicrob Chemother 2011; 66:1510-1513.

4. Voulgari E, Gartzonika C, Vrioni G, et al. The Balkan region: NDM-1-producing Klebsiella pneumoniae ST11 clonal strain causing outbreaks in Greece. J Antimicrob Chemother 2014;69: 2091-2097. 
5. Tsakris A, Poulou A, Pournaras S, et al. A simple phenotypic method for the differentiation of metallo-beta-lactamases and class A KPC carbapenemases in Enterobacteriaceae clinical isolates. J Antimicrob Chemother 2010;65:1664-1671.

6. Nordmann P, Poirel L, Carrër A, Toleman MA, Walsh TR. How to detect NDM-1 producers. J Clin Microbiol 2011;49:718-721.

7. Meletis G, Vavatsi N, Exindari M, et al. Accumulation of carbapenem resistance mechanisms in VIM-2-producing Pseudomonas aeruginosa under selective pressure. Eur J of Clin Microbiol Infect Dis 2014;33:253-258.

8. Meletis G, Chatzidimitriou D, Malisiovas N. Double-and multi-carbapenemase-producers: the excessively armored bacilli of the current decade. Eur J of Clin Microbiol Infect Dis 2015;34: 1487-1493.

9. Poirel L, Potron A, Nordmann P. OXA-48-like carbapenemases: the phantom menace. J Antimicrob Chemother 2012;67:1597-1606.

E R R A T U M

Research Methods in Healthcare Epidemiology and Antimicrobial Stewardship: Use of Administrative and Surveillance Databases - ERRATUM

In this issue of the journal, the article by Drees et al. ${ }^{1}$ was incorrectly published as an Original Article, rather than a SHEA White Paper. The article type has since been corrected, and the editors and publisher apologize for this error.

\section{REFERENCE}

1. Drees M, Gerber JS, Morgan DJ, Lee GM. Research methods in healthcare epidemiology and antimicrobial stewardship: use of administrative and surveillance databases. Infect Control Hosp Epidemiol 2016;37:1278-1287. 